\title{
Stereotactic body radiation therapy (SBRT) for patients with stage I non-small cell lung cancer is applicable to more tumors than sublobar resection
}

\author{
Andrew J. Song ${ }^{\wedge}$, Nathaniel Evans ${ }^{2}$, Scott Cowan ${ }^{2}$, Jenny Guo ${ }^{3}$, Tingting Zhan ${ }^{4}$, Bo Lu ${ }^{1}$, \\ Maria Werner-Wasik ${ }^{1}$ \\ ${ }^{1}$ Department of Radiation Oncology, Sidney Kimmel Cancer Center, Thomas Jefferson University, Philadelphia, PA, USA; ${ }^{2}$ Department of \\ Surgery, Division of Thoracic Surgery, Thomas Jefferson University, Philadelphia, PA, USA; ${ }^{3}$ Sidney Kimmel Medical College at Thomas Jefferson \\ University, Philadelphia, PA, USA; ${ }^{4}$ Department of Pharmacology \& Experimental Therapeutics, Division of Biostatistics, Thomas Jefferson \\ University, Philadelphia, PA, USA \\ Contributions: (I) Conception and design: M Werner-Wasik, AJ Song, N Evans, S Cowan; (II) Administrative support: AJ Song, J Guo; (III) Provision \\ of study materials or patients: M Werner-Wasik, B Lu; (IV) Collection and assembly of data: AJ Song, J Guo; (V) Data analysis and interpretation: M \\ Werner-Wasik, AJ Song, T Zhan, N Evans, S Cowan, B Lu; (VI) Manuscript writing: All authors; (VII) Final approval of manuscript: All authors. \\ Correspondence to: Maria Werner-Wasik, MD. 111 S $11^{\text {th }}$ Street, Suite G-301, Philadelphia, PA 19107, USA. Email: Maria.Werner-Wasik@Jefferson.edu.
}

Background: Virtually all patients with medically inoperable stage I non-small cell lung cancer (NSCLC) can receive stereotactic body radiation therapy. However, the percentage of such patients in whom sublobar resection is technically feasible is unknown. This discrepancy can confound clinical trial eligibility and designs comparing stereotactic body radiation therapy $v s$. sublobar resection.

Methods: A total of 137 patients treated with stereotactic body radiation therapy for lung lesions (3/201311/2017) underwent retrospective review. Diagnostic CT chest and PET/CT images, stereotactic body radiation therapy dates, and demographic data were collected on 100 of 137 patients. Two experienced boardcertified thoracic surgeons independently reviewed anonymized patients' pre-stereotactic body radiation therapy diagnostic imaging and completed a custom survey about the technical feasibility of sublobar resection for each patient. Interrater agreement was measured using Cohen's kappa coefficient by bootstrap methodology. Summary statistics were performed for baseline demographics and tumor characteristics.

Results: Of the 100 patients, 57\% were female, with median age of 75 years (range, 52-95 years) and Karnofsky Performance Status of 80 (range, 40-100). Most patients (61\%) had Stage IA1, T1a tumors. For interrater agreement analysis, one patient was removed from each cohort due to inability to locate tumor on images, leaving 98 patients analyzed. Comparing Surgeon \#1 vs. Surgeon \#2, 64 (65.3\%) vs. 69 (70.3\%) of tumors were thought eligible for sublobar resection, respectively $(\kappa=0.414)$.

Conclusions: Stereotactic body radiation therapy for stage I NSCLC is applicable to more tumors than sublobar resection, with $\sim 30-35 \%$ of stereotactic body radiation therapy patients unable to undergo sublobar resection assessed by pretreatment diagnostic imaging based on technical grounds. This study illustrates that clinical trials comparing stereotactic body radiation therapy $v s$. sublobar resection are limited to only a subpopulation of patients with stage I NSCLC.

Keywords: Stereotactic body radiation therapy (SBRT); stereotactic ablative radiotherapy (SAbR); sublobar resection; wedge resection; segmentectomy; early stage non-small cell lung cancer (early stage NSCLC)

Submitted May 22, 2020. Accepted for publication Dec 30, 2020.

doi: $10.21037 /$ jtd-20-2001

View this article at: http://dx.doi.org/10.21037/jtd-20-2001

^ ORCID: 0000-0001-8386-4397.

(C) Journal of Thoracic Disease. All rights reserved. 


\section{Introduction}

Stereotactic body radiation therapy (SBRT), also known as stereotactic ablative radiotherapy ( $\mathrm{SAbR}$ ), is often offered as an alternative to surgery for early stage non-small cell lung cancer (NSCLC) patients who are medically inoperable or who decline surgery (1). Virtually all of these patients can receive SBRT despite their medical shortcomings. SBRT has been proven to have a high rate of local control ( 90$95 \%$ ) with limited toxicity, even with long-term follow-up, with the most common recurrences occurring regionally or distantly (2). In a pooled analysis of the STARS and ROSEL trials, which looked at operable Stage I NSCLC patients, SBRT was found to have a higher overall survival of $95 \%$ at 3 years compared to patients who had received lobectomy and mediastinal lymph node dissection at $75 \%$, with similar recurrence free survival (3). Patients who had received surgery had more Grade 3 or higher events than the SBRT arms (44\% vs. 10\%).

An ongoing phase 3 trial, STABLE-MATES, is looking to determine if this overall survival difference will be observed in a larger patient population, when comparing head-to-head sublobar resection vs SBRT with 54 Gy in 3 fractions for early stage peripheral lesions (4). One of the inclusion criteria is whether a patient's tumor is in a location that will allow for a sublobar resection. Sublobar resection, performed as a wedge resection or segmentectomy, is more akin to SBRT, with recent studies suggesting similar clinical outcomes to lobectomy in certain contexts when accounting for tumor size and location (5-7). This criterion raises an interesting question. What proportion of patients eligible for SBRT are not eligible for sublobar resection due to technical inability to resect the tumor? To date, there is no published literature quantifying this specific parameter which could potentially change clinical trial design and patient eligibility regarding these modalities in the future, as well as specific recommendation for clinical practice guidelines.

We report a novel assessment of early stage NSCLC patients eligible for and treated with SBRT, but who are not amenable to sublobar resection due to technical considerations.

We present the following article in accordance with the STROBE reporting checklist (available at http://dx.doi. org/10.21037/jtd-20-2001).

\section{Methods}

The study was conducted in accordance with the Declaration of Helsinki (as revised in 2013). The study was approved by the Institutional Review Board at Thomas Jefferson University (Philadelphia, PA; IRB Control \# 17D.549) and individual consent for this retrospective analysis was waived.

We identified 137 consecutive patients treated at our institution with SBRT for lung lesions from March 2013 to November 2017. Of these patients, 37 patients were removed from analysis for the following reasons: 33 patients received SBRT for lung metastases and 4 lacked preSBRT imaging records. For the remaining 100 patients, we collected diagnostic CT chest images, PET/CT images, SBRT treatment details, and demographic data (Figure 1). Staging was reported per AJCC $7^{\text {th }}$ edition.

Two experienced board-certified thoracic surgeons, with 7 and 11 years of practice and performing 80 to 100 surgeries per year for stage I NSCLC at time of study, independently reviewed each patient's anonymized preSBRT diagnostic CT chest scans along with corresponding PET/CT images when available. With each patient review, the thoracic surgeons filled out a customized survey which was created with input from radiation oncologists and thoracic surgeons to evaluate technical feasibility of a sublobar resection of primary lung lesions (Figure 2). This survey asked questions on the following:

(I) Ability to identify the lung tumor in question (yes/no);

(II) Technical feasibility of any sublobar resection (yes/no);

(III) Technical feasibility of wedge resection (yes/no);

(IV) Technical feasibility of segmentectomy (yes/no).

Surgeons were asked to provide the best answer to each of the questions. If the surgeons answered "no" to question \#III, they were further prompted to provide reasons for not being able to perform wedge resection including:

i. The tumor is too deep within the lung;

ii. Inadequate margin due to tumor size;

iii. Inadequate margin due to tumor location;

iv. The tumor is too small to be palpable;

v. Other (free-form response).

Similarly, if the surgeons answered "no" to question \#IV, then reasons against segmentectomy included:

i. The tumor is not clearly confined to one segment;

ii. Inadequate margin due to tumor size;

iii. Inadequate margin due to tumor location;

iv. Segmental anatomy is not clear on imaging;

v. Other (free-form response).

\section{Statistical analysis}

Interrater agreement between the surgeons was measured 


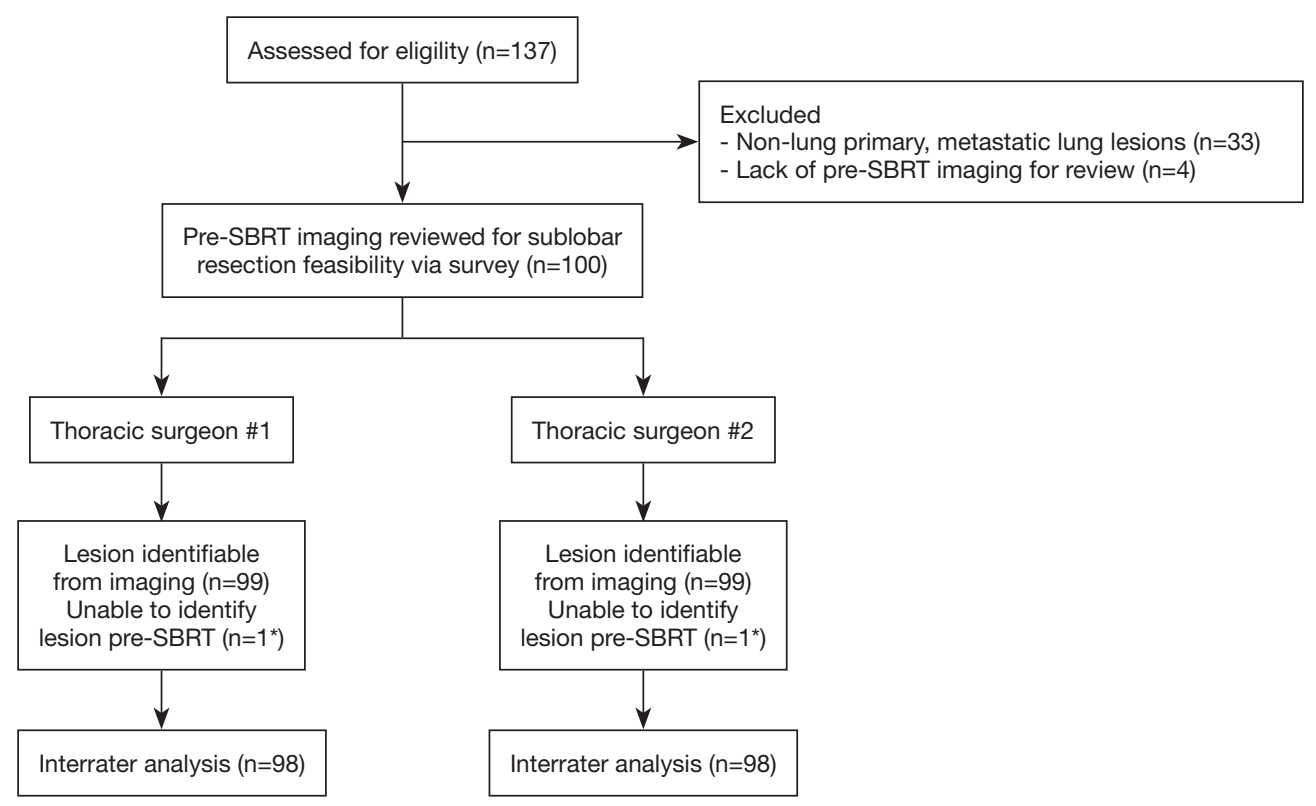

Figure 1 CONSORT diagram. *, each surgeon was unable to identify one lesion of interest on pre-SBRT imaging for one patient. These were different patients, so for interrater analysis, both of these patients were excluded.

using Cohen's kappa coefficient by bootstrap methodology (8). Kappa coefficient $(\kappa)$ ranges were determined as follows (9):

i. $\quad \kappa \leq 0$ as indicating no agreement;

ii. $\quad 0.01 \geq \kappa \leq 0.20$ as none to slight;

iii. $\quad 0.21 \geq \kappa \leq 0.40$ as fair;

iv. $\quad 0.41 \geq \kappa \leq 0.60$ as moderate;

v. $\quad 0.61 \geq \kappa \leq 0.80$ as substantial and;

vi. $\quad 0.81 \geq \kappa \leq 1.00$ as almost perfect agreement.

For reasons against technical feasibility of wedge resection (question \#IV) or segmentectomy (question \#VI), we examined interrater agreement analysis for patients where both Surgeon \#1 and Surgeon \#2 were in consensus that the lesion was not amenable to wedge resection or segmentectomy. In the event that a surgeon provided multiple responses for a lesion, interrater analysis was determined through partial agreement. For example, if Surgeon \#1 responds 'i' and Surgeon \#2 responds 'i/ii', we consider the second response to be ' $i$ ' and thus qualify as a match to the first response. On the other hand, If Surgeon \#1 responds ' $i$ ' and Surgeon \#2 responds 'ii/iii', then we would consider the second response to be 'ii' (or 'iii') and not qualify as a match to the first answer.

Summary statistics were performed for baseline demographics, tumor characteristics, and surgeon-provided reasons against sublobar resection techniques. Statistics were analyzed with $\mathrm{R}$ (version 3.5.1).

\section{Results}

\section{Patient demographics}

Of the 100 patients, $57 \%$ were female and $43 \%$ were male. Median age at SBRT was 75 years (range, 52-95 years) and median KPS was 80 (range, 40-100). The majority of patients $(61 \%)$ had stage IA1, T1a tumors, followed by stage IA2, T1b (22\%), and stage IB, T2a (17\%).

\section{Individual surgeon responses}

Surgeon \#1 identified lesions from 99 patients for the available pre-SBRT imaging. Surgeon \#2 also identified 99 lesions from the pre-SBRT imaging. However, these lesions were in separate patients, so after these two patients were removed, 98 patients underwent further analysis.

According to Surgeon \#1, of these 98 patients, 64 (65.3\%) were amenable to sublobar resection. Of these, $64(100 \%)$ had the option of wedge resection as feasible while 42 (65.6\%) were considered feasible for segmentectomy. The most common reason against wedge resection was "depth of tumor in lung" for 22 patients (64.7\%). The most common reason against segmentectomy was "inadequate margin due to tumor location" and "segmental anatomy not clear on imaging", both with 23 responses (41.1\%).

For Surgeon \#2, 69 (70.4\%) of tumors were amenable 
1. Are you able to identify the lung tumor in question? a. Yes

b. No

2. Is this tumor amenable to a sublobar resection ? a. Yes

b. No

3. If yes, can wedge resection be performed? a. Yes

b. No

4. If not wedge, why?

a. The tumor is too deep within the lung

b. Inadequate margin due to tumor size

c. inadequate margin due to tumor location

d. The tumor is too small to be palpable

e. Other: fill in the blank

5. If yes, can segmentectomy be performed?

$$
\text { a. Yes }
$$

b. No

6. If not segmentectomy, why?
a. The tumor is not clearly confined to one segment
b. Inadequate margin due to tumor size
c. inadequate margin due to tumor location
d. segmental anatomy is not clear on imaging
e. Other: __ fill in the blank

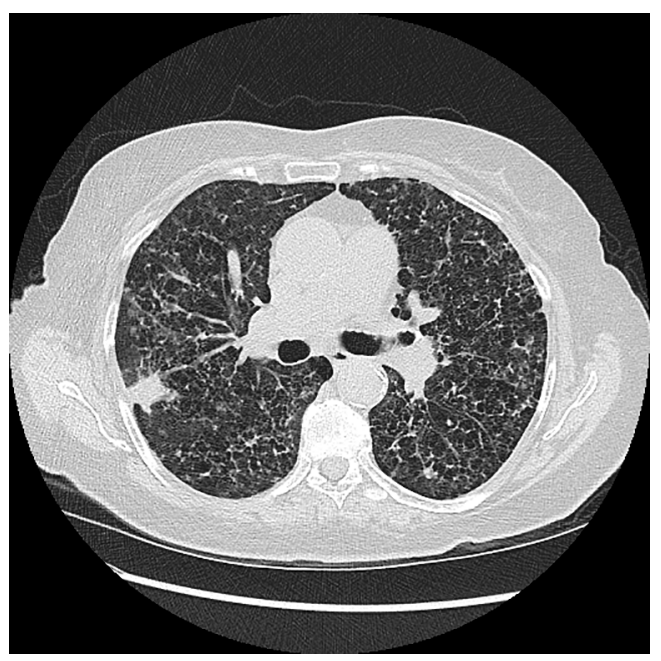

Figure 2 Thoracic surgeon survey with sample image.

to sublobar resection. Of these, $58(84 \%)$ had the option of wedge resection as feasible while $42(62.3 \%)$ were considered feasible for segmentectomy. When prompted for reasons against a sublobar resection, at times Surgeon \#2 provided multiple responses (e.g., 'A' and 'B'), with 10 patients (24 responses) for wedge resection and 14 patients (29 responses) for segmentectomy, so all of these were reported. The most common reason against wedge resection for Surgeon \#2 was also "depth of tumor in lung" for 32 patients $(80 \%)$. The most common reason against segmentectomy was different, with "tumor not clearly confined to one segment" in 44 patients (80\%). Please see Figure 3 for detailed response breakdown.

\section{Interrater agreement analysis}

For interrater agreement analysis, one patient each was removed from each set of surgeon responses due to a response of "inability to identify the tumor" on images. These patients were different, thus leaving 98 unique patients for analysis comparing responses between Surgeon \#1 and Surgeon \#2.

When comparing technical feasibility for sublobar resection, there was moderate interrater agreement $(\kappa=0.414)$. For wedge resection feasibility, interrater agreement was slightly higher $(\kappa=0.473)$. For segmentectomy, the interrater agreement was fair $(\kappa=0.225)$.

When examining reported reasons against wedge resection, there was moderate interrater agreement $(\kappa=0.431)$. However, for reasons against segmentectomy, there was only slight agreement $(\kappa=0.052)$. See Table 1 .

\section{Discussion}

Our results suggest that approximately $30-35 \%$ of patients who are able to receive SBRT are not able to undergo sublobar resection, based on technical considerations from pretreatment diagnostic imaging. This represents a subpopulation of early stage NSCLC patients that is often overlooked in the current treatment paradigm. The proportion of patients deemed eligible for wedge resection ( $60 \%)$ was higher than segmentectomy ( $40 \%)$, with moderate to fair interrater agreement. Agreement on reasons against segmentectomy was also relatively lower than that on wedge resection.

Although lobectomy is the standard of care for medically operable patients (10), there exists a subset of patients in whom sublobar resection may be considered. The NCCN guidelines define this as patients with poor pulmonary reserve, major comorbidities, and/or peripheral nodule $\leq 2 \mathrm{~cm}$ in size with $\geq 50 \%$ ground-glass appearance or long doubling time (1). These same patients are oftentimes also eligible for SBRT as it is felt that SBRT has minimal risks associated with treatment of such patients with resulting stable pulmonary function post-treatment. Additionally, more recent studies have shown that sublobar resection can be comparable to lobectomy for clinical outcomes after adjusting for confounders and also factoring in 


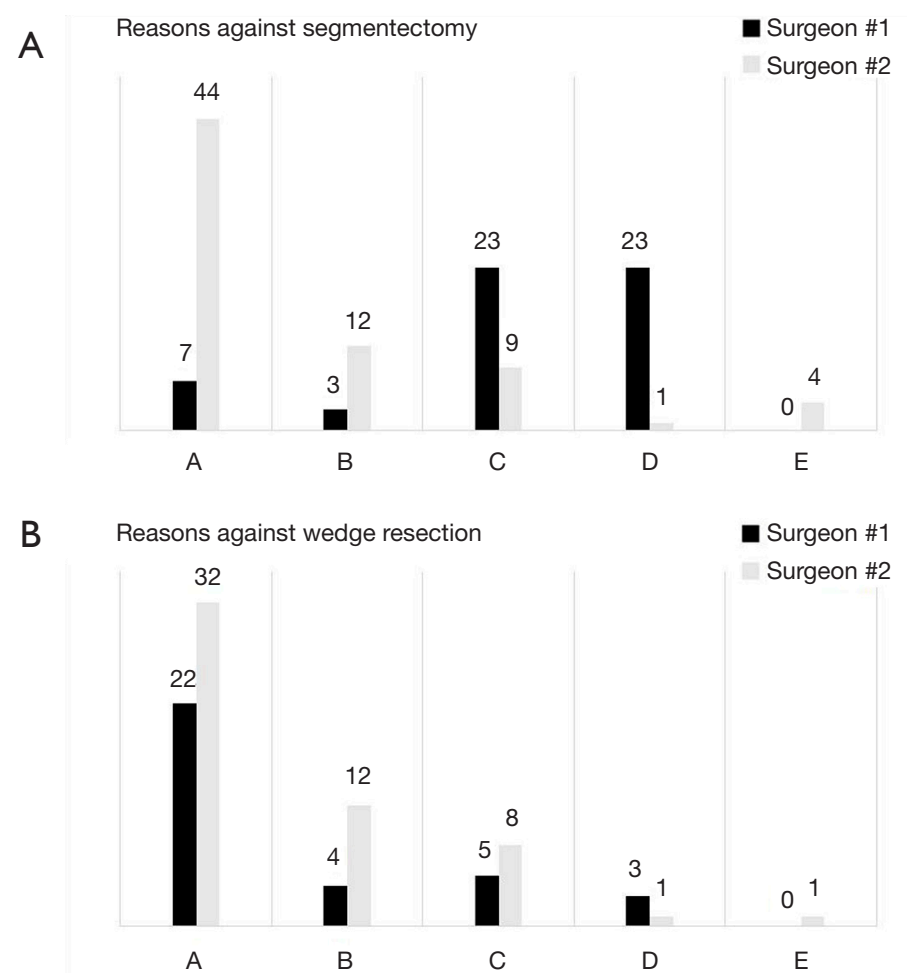

Figure 3 Individual surgeon survey responses. (A) A, the tumor is not clearly confined to one segment; B, inadequate margin due to tumor size; C, inadequate margin due to tumor location; D, segmental anatomy is not clear on imaging; E, other (free-form response). Surgeon \#2 provided multiple responses for some patients, thus these have all been compiled leading to more than 55 responses (total 70). (B) A, the tumor is too deep within the lung; B, inadequate margin due to tumor size; C, inadequate margin due to tumor location; D, the tumor is too small to be palpable; E, other (free-form response). Surgeon \#2 provided multiple responses for some patients, thus these have all been compiled leading to more than 40 responses (total 54).

Table 1 Interrater agreement analysis

\begin{tabular}{lccc}
\hline Technical feasibility & Kappa coefficient $(\kappa)$ & Magnitude & $\mathrm{P}$ value \\
\hline Sublobar resection & 0.414 & Moderate & $<0.001$ \\
Wedge resection & 0.473 & Moderate & $<0.001$ \\
Segmentectomy & 0.225 & Fair & 0.027 \\
$\begin{array}{l}\text { Reasons against } \\
\text { wedge resection }\end{array}$ & 0.431 & Moderate & 0.001 \\
$\begin{array}{l}\text { Reasons against } \\
\text { segmentectomy }\end{array}$ & 0.052 & Slight & 0.331 \\
\hline
\end{tabular}

size and location of lesions while preserving pulmonary function (7,11-15). An earlier meta-analysis of stage I NSCLC patients showed no overall survival differences between these surgical techniques at 1,3 , and 5 years with a subsequent, separate meta-analysis also showing no differences in disease-free and overall survival for patients intentionally selected for sublobar resection when compared to their lobectomy counterparts with small, peripheral lesions $(6,12)$. SBRT has also been evaluated for early stage peripheral lesions in medically operable patients in the setting of a single-arm phase II clinical trial, RTOG 0618, with 26 patients evaluated and achieving relatively high local control (96\%) and low rates of surgical salvage and adverse events while preserving pulmonary function (16). SBRT is also more cost-effective for marginally operable stage I NSCLC patients when compared to either wedge resection or lobectomy (17). Markov modeling of medically operable patients stratified by smoking history suggested SBRT was also similar to surgery when comparing quality-adjusted life years (QALY) (18). Through a propensity scorematched analysis of National Cancer Database, an increased mortality risk associated with more extensive surgery and older age were found when compared to SBRT. Although SBRT has been traditionally associated with treatment of 
peripheral lung lesions due to early experiences with excess toxicity for centrally located tumors (19), our improved understanding for dosing and constraints for organs at risk has led to more patients with centrally located tumors to now be eligible for SBRT. A recent phase I/II study, RTOG 0813, determined that central tumors could be treated comparably to peripheral tumors with a MTD of 12 Gy per fraction (20). In recent years, the national trend away from lobectomy and towards sublobar resection and SBRT makes these findings more pertinent (21). Therefore, we believe that our comparison of these two treatment modalities is important for being taken into consideration for managing a subpopulation of early stage NSLC patients.

Our study is limited to two board certified thoracic surgeons and could be further improved with additional participation. In addition, while our study does analyze strengths of interrater agreement, we lack intrarater reliability. The latter could provide additional insights on the consistency of assessment for which lesions are deemed resectable by an individual. External validation of the customized survey will introduce additional data for interrater agreement analysis.

Although our surgeons had moderate interrater agreement regarding ineligibility for wedge resection and corresponding reasons against wedge resection, the interrater agreement for segmentectomy was relatively lower with fair interrater agreement for ineligibility and even lower for the reasons against segmentectomy. This is an interesting finding, as segmentectomy is a more complex procedure than wedge resection, with the latter a nonanatomic resection. This disparity is likely multifactorial, including surgeon-specific training, experience and preferential practice as well as patient anatomies and tumor location, size, and appearance.

Current practice guidelines for early stage NSCLC are available from several organizations, including the American Society for Radiation Oncology, European Society for Medical Oncology, National Comprehensive Cancer Network, and Spanish Society of Medical Oncology. These guidelines tend to favor lobectomy, followed by sublobar resection, and lastly by SBRT $(1,22-24)$. For medically operable patients, SBRT is recommended against unless in the setting of a clinical trial. Part of this rationale is that surgery can provide additional nodal pathology for staging purposes so that there remains the risk of under-staging due to occult disease, with PET/CT scans having a false-negative rate approximately $10-15 \%$ for clinical stage I disease (25-27). However, a meta-analysis of over 1,000 patients from 10 studies, examined the negative predictive value of PET/CT and found NPV as high as $94 \%$ for mediastinal metastases for T1 disease (28). Therefore, we believe that for some early stage NSCLC patients who are not resectable candidates or who will elect to decline surgery, the benefits from invasive nodal staging should be balanced by the risks and costs associated with such a strategy. There are several options for mediastinal staging, including no invasive mediastinal staging, endobronchial ultrasoundguided transbronchial needle aspiration (EBUS-TBNA), and mediastinoscopy. When comparing these options for QALY in a single payer system, there were relatively small realworld disparities between these modalities (5.80 vs. $5.87 \mathrm{vs}$. 5.86 QALYs, respectively), resulting in approximately 3-3.5 weeks' difference of optimal health (29). When comparing the incremental cost-effectiveness ratios (ICER) for these mediastinal staging options, for $\mathrm{cN} 0$ patients with low risk $(<2.5 \%)$ of $\mathrm{pN} 2$ involvement, invasive mediastinal staging is not cost-effective with a ICER of $\sim \$ 20,016.68 / \mathrm{QALY}$ for EBUS-TBNA alone and $\sim \$ 1,076,163.63 /$ QALY for EBUSTBNA with mediastinoscopy. In addition, we should also consider the operative mortality associated with surgical resection, ranging from $1.1 \%$ for wedge resection, $1.8 \%$ for segmentectomy, and $2.0 \%$ for lobectomy patients (30). These elements should be factored in the decision-making process when managing early stage NSCLC patients, especially for those who may be borderline medically operable or are not optimal candidates for resectability.

Clinical trials attempting to compare surgery and SBRT have faced barriers with accrual and questions of equipoise $(3,31)$. Ongoing phase III trials, such as the VALOR trial (32) comparing lobectomy or segmentectomy with mediastinal lymph node sampling (adjuvant chemotherapy for positive lymph nodes) vs SBRT and the SABRTOOTHv1 trial (33) in the UK which compares sublobar resection +/- intraoperative brachytherapy vs. SBRT, will help elucidate the value of SBRT in medically operable patients in the setting of anatomic and nonanatomic resections as well as utility of brachytherapy with sublobar resection. Future clinical trials hoping to compare these treatment modalities may take into consideration that a subpopulation of patients will be considered medically operable but are ineligible for sublobar resection due to technical reasons, which will affect screening and potential enrollment success. In addition, we believe that prior clinical trials would have excluded such patients from enrollment and analysis, which could lead to a bias in patient selection and ultimately affecting the outcomes of comparing these modalities.

Our study is the first of its kind to report a quantifiable 
estimate on the proportion of early stage NSCLC patients who are treatable with SBRT, but are not appropriate for sublobar resection based on technical criteria. Results from prior clinical trials may need to be qualified by extrapolating outcomes only to patients who are both medically and technically operable. This specific subpopulation of patients should be considered when developing practice guidelines for care in early stage NSCLC patients as well as for designing eligibility criteria and screening for clinical trials comparing sublobar resection with SBRT.

\section{Acknowledgments}

The authors would like to thank Lilya Babinsky for her assistance in retrieving data.

Funding: None.

\section{Footnote}

Reporting Checklist: The authors have completed the STROBE reporting checklist. Available at http://dx.doi. org/10.21037/jtd-20-2001

Data Sharing Statement: Available at http://dx.doi. org/10.21037/jtd-20-2001

Conflicts of Interest: All authors have completed the ICMJE uniform disclosure form (available at http://dx.doi. org/10.21037/jtd-20-2001). The authors have no conflicts of interest to declare.

Ethical Statement: The authors are accountable for all aspects of the work in ensuring that questions related to the accuracy or integrity of any part of the work are appropriately investigated and resolved. The study was conducted in accordance with the Declaration of Helsinki (as revised in 2013). The study was approved by the Institutional Review Board at Thomas Jefferson University (Philadelphia, PA; IRB Control \# 17D.549) and individual consent for this retrospective analysis was waived.

Open Access Statement: This is an Open Access article distributed in accordance with the Creative Commons Attribution-NonCommercial-NoDerivs 4.0 International License (CC BY-NC-ND 4.0), which permits the noncommercial replication and distribution of the article with the strict proviso that no changes or edits are made and the original work is properly cited (including links to both the formal publication through the relevant DOI and the license). See: https://creativecommons.org/licenses/by-nc-nd/4.0/.

\section{References}

1. Ettinger DS, Wood DE, Chair V, et al. NCCN Guidelines Version 3.2020 Non-Small Cell Lung Cancer. Available online: https://www.nccn.org/professionals/physician_gls/ pdf/nscl.pdf, accessed on Feb 15th, 2020.

2. Sun B, Brooks ED, Komaki RU, et al. 7-year follow-up after stereotactic ablative radiotherapy for patients with stage I non-small cell lung cancer: Results of a phase 2 clinical trial. Cancer 2017;123:3031-9.

3. Chang JY, Senan S, Paul MA, et al. Stereotactic ablative radiotherapy versus lobectomy for operable stage I nonsmall-cell lung cancer: a pooled analysis of two randomised trials. Lancet Oncol 2015;16:630-7.

4. JoLT-Ca Sublobar Resection (SR) Versus Stereotactic Ablative Radiotherapy (SAbR) for Lung Cancer. Available online: https://clinicaltrials.gov/ct2/show/NCT02468024 (accessed 20 December 2019).

5. Yendamuri S, Sharma R, Demmy M, et al. Temporal trends in outcomes following sublobar and lobar resections for small $(\leq 2$ $\mathrm{cm})$ non-small cell lung cancers--a Surveillance Epidemiology End Results database analysis. J Surg Res 2013;183:27-32.

6. Cao C, Gupta S, Chandrakumar D, et al. Meta-analysis of intentional sublobar resections versus lobectomy for early stage non-small cell lung cancer. Ann Cardiothorac Surg 2014;3:134-41.

7. El-Sherif A, Gooding WE, Santos R, et al. Outcomes of sublobar resection versus lobectomy for stage I non-small cell lung cancer: a 13-year analysis. Ann Thorac Surg 2006;82:408-15; discussion 415-6.

8. Altman DG. Practical statistics for medical research. Chapman and Hall, https://www.crcpress.com/ Practical-Statistics-for-Medical-Research/Altman/p/ book/9780412276309 (1991, accessed 3 January 2019).

9. Mchugh ML. Interrater reliability: the kappa statistic Interrater reliability: the kappa statistic Interrater reliability: the kappa statistic Page 2 of 14 . Biochem Medica 2012;22:276-82.

10. Ginsberg RJ, Rubinstein LV. Randomized trial of lobectomy versus limited resection for T1 N0 non-small cell lung cancer. Lung Cancer Study Group. Ann Thorac Surg 1995;60:615-22; discussion 622-3.

11. Keenan RJ, Landreneau RJ, Maley RH Jr, et al. Segmental resection spares pulmonary function in patients with stage I lung cancer. Ann Thorac Surg 2004;78:228-33. 
12. Nakamura H, Kawasaki N, Taguchi M, et al. Survival following lobectomy vs limited resection for stage I lung cancer: a meta-analysis. Br J Cancer 2005;92:1033-7.

13. Okada M, Koike T, Higashiyama M, et al. Radical sublobar resection for small-sized non-small cell lung cancer: a multicenter study. J Thorac Cardiovasc Surg 2006;132:769-75.

14. De Giacomo T, Di Stasio M, Diso D, et al. Sub-lobar lung resection of peripheral T1N0M0 NSCLC does not affect local recurrence rate. Scand J Surg 2009;98:225-8.

15. Zheng X, Schipper M, Kidwell K, et al. Survival outcome after stereotactic body radiation therapy and surgery for stage I non-small cell lung cancer: a meta-analysis. Int J Radiat Oncol Biol Phys 2014;90:603-11.

16. Timmerman RD, Paulus R, Pass HI, et al. Stereotactic Body Radiation Therapy for Operable Early-Stage Lung Cancer: Findings From the NRG Oncology RTOG 0618 Trial. JAMA Oncol 2018;4:1263-6.

17. Shah A, Hahn SM, Stetson RL, et al. Cost-effectiveness of stereotactic body radiation therapy versus surgical resection for stage I non-small cell lung cancer. Cancer 2013;119:3123-32.

18. Louie AV, Rodrigues G, Hannouf M, et al. Stereotactic body radiotherapy versus surgery for medically operable Stage I non-small-cell lung cancer: a Markov model-based decision analysis. Int J Radiat Oncol Biol Phys 2011;81:964-73.

19. Timmerman R, McGarry R, Yiannoutsos C, et al. Excessive toxicity when treating central tumors in a phase II study of stereotactic body radiation therapy for medically inoperable early-stage lung cancer. J Clin Oncol 2006;24:4833-9.

20. Bezjak A, Paulus R, Gaspar LE, et al. Safety and Efficacy of a Five-Fraction Stereotactic Body Radiotherapy Schedule for Centrally Located Non-Small-Cell Lung Cancer: NRG Oncology/RTOG 0813 Trial. J Clin Oncol 2019;37:1316-25.

21. McMurry TL, Shah PM, Samson P, et al. Treatment of stage I non-small cell lung cancer: What's trending? J Thorac Cardiovasc Surg 2017;154:1080-7.

22. Videtic GMM, Donington J, Giuliani M, et al. Stereotactic body radiation therapy for early-stage non-small cell lung cancer: Executive Summary of an ASTRO Evidence-Based Guideline. Pract Radiat Oncol 2017;7:295-301.

23. Postmus PE, Kerr KM, Oudkerk M, et al. Early and locally advanced non-small-cell lung cancer (NSCLC): ESMO Clinical Practice Guidelines for diagnosis, treatment and follow-up. Ann Oncol 2017;28:iv1-21.

24. Majem M, Juan O, Insa A, et al. SEOM clinical guidelines for the treatment of non-small cell lung cancer (2018). Clin Transl Oncol 2019;21:3-17.

25. Gómez-Caro A, Boada M, Cabañas M, et al. False- negative rate after positron emission tomography/ computer tomography scan for mediastinal staging in cI stage non-small-cell lung cancer. Eur J Cardiothorac Surg 2012;42:93-100.

26. Al-Sarraf N, Aziz R, Gately K, et al. Pattern and predictors of occult mediastinal lymph node involvement in non-small cell lung cancer patients with negative mediastinal uptake on positron emission tomography. Eur J Cardiothorac Surg 2008;33:104-9.

27. Robinson EM, Ilonen IK, Tan KS, et al. Prevalence of Occult Peribronchial N1 Nodal Metastasis in Peripheral Clinical N0 Small ( $\leq 2 \mathrm{~cm})$ Non-Small Cell Lung Cancer. Ann Thorac Surg 2020;109:270-6.

28. Wang J, Welch K, Wang L, et al. Negative predictive value of positron emission tomography and computed tomography for stage T1-2N0 non-small-cell lung cancer: a meta-analysis. Clin Lung Cancer 2012;13:81-9.

29. Czarnecka-Kujawa K, Rochau U, Siebert U, et al. Costeffectiveness of mediastinal lymph node staging in non-small cell lung cancer. J Thorac Cardiovasc Surg 2017;153:1567-78.

30. Gulack BC, Yang CJ, Speicher PJ, et al. A Risk Score to Assist Selecting Lobectomy Versus Sublobar Resection for Early Stage Non-Small Cell Lung Cancer. Ann Thorac Surg 2016;102:1814-20.

31. Fernando HC, Timmerman R. American College of Surgeons Oncology Group Z4099/Radiation Therapy Oncology Group 1021: a randomized study of sublobar resection compared with stereotactic body radiotherapy for high-risk stage I non-small cell lung cancer. J Thorac Cardiovasc Surg 2012;144:S35-8.

32. Veterans Affairs Lung Cancer Surgery Or Stereotactic Radiotherapy. Available online: https://clinicaltrials.gov/ ct2/show/NCT02984761 (accessed 9 February 2020).

33. A Study to Determine the Feasibility and Acceptability of Conducting a Phase III Randomised Controlled Trial Comparing Stereotactic Ablative Radiotherapy With Surgery in paTients With Peripheral Stage I nOn-small Cell Lung Cancer cOnsidered Higher Risk of Complications From Surgical Resection. Available online: https:/clinicaltrials.gov/ ct2/show/NCT02629458 (accessed 9 February 2020).

Cite this article as: Song AJ, Evans N, Cowan S, Guo J, Zhan T, Lu B, Werner-Wasik M. Stereotactic body radiation therapy (SBRT) for patients with stage I non-small cell lung cancer is applicable to more tumors than sublobar resection. J Thorac Dis 2021;13(3):1576-1583. doi: 10.21037/jtd-20-2001 